\title{
DE PLANTIS LEGIONENSIBUS. NOTULA XI
}

\author{
Luis HERRERO CEMBRANOS, Marta E. GARCIA GONZALEZ \\ \& Angel PENAS
}

\begin{abstract}
RESUMEN: En el presente trabajo se aportan datos corológicos y ecológicos sobre algunas fanerógamas, que se citan por primera vez para la flora leonesa, tales como: Atriplex patula L., Lepidium latifolium L., Lotus tenuis Waldst. E Kit. ex Willd. var. tenuis, Hypericum androsaemum L., Thymus $x$ sennenii Pau, Verbascum blattaria L., Carduus bourgeanus Boiss. E Reuter, Picnomon acarna (L.) Cass., Onopordum nervosum Boiss., Centaurea aspera L. De otras se indican nuevas localidades.
\end{abstract}

SUMMARY: In the present paper, some chorologic and ecologic facts about several phanerogams recorded for the first time within the flora of León are given. They are: Atriplex patula L., Lepidium latifolium L., Lotus tenuis Waldst. E Kit. ex Willd. var. tenuis, Hypericum androsaemum L., Thymus $x$ sennenii Pau, Verbascum blattaria L., Carduus bourgeanus Boiss. E Reuter, Picnomon acarna (L.) Cass., Onopordum nervosum Boiss., Centaurea aspera L. On the other hand, we indicate new localities.

La presente nota floristica es fruto de diversas prospecciones botánicas en la provincia de León, cuyos materiales se hallan depositados en el herbario de la Facultad de Biología de León. De cada taxon se indican las coordenadas UTM, en cuadriculas de $10 \mathrm{Km}$ de 1 ado.

Atriplex patula L., Sp. Pl. 1053 (1753)

LEON: Monte del Duque (Valderas) 30TTM98. 21-IX-1986, (LEB 30972). Leg.: A. Penas, M.E. García \& L. Herrero. En comunidades de la Ruderali-Secalietea $\mathrm{Br} . \mathrm{Bl}$. 1936.

Especie de la que no conocemos citas provinciales y que Merino (1905) menciona de la costas de Pontevedra y La Coruña, mientras que Lázaro Ibiza (1920) y Willkomm \& Lange (1870) la consideran más o menos abundante por toda España.

Polycarpon tetraphyllum (L.) L., Syst. Nat. ed. 10, 2:881 (1759)

LEON: Castropodame 29TQHO1. 31-V-1986, (LEB 32099). Leg.: A. Penas, M.E. García \& L. Herrero.

La única mención provincial que conocemos para este taxon es la que recoge Colmeiro (1886) de Lange en Villafranca del Bierzo. Aportamos pues su segunda cita para la provincia. 
Spergularia segetalis (L.) G. Don fil., Gen. Syst. 1:425 (1831)

LEON: Valverde Enrique 30TUM18. 21-V-1986, (LEB 32154) y Lugán $30 T U N 03$. 5-VI-1983, (LEB 30973). Leg.: A. Penas, M.E. García \& L. Herrero.

La única mención provincial que conocemos para este taxon es la que recoge Colmeiro (1886) de Lange en Villafranca del Bierzo. Aportamos pues su segunda cita para la provincia.

R. Carbo, M. Mayor, J. Andrés \& J.M. Losa (1977) la señalan para la provincia de Llamas de Rueda (Sector Leonés) y Herreros de Jamuz (Sector Orensano-Sanabriense) sin que exista material de herbario. Confirmamos su presencia en León.

Lepidium latifolium L., Sp. Pl. 644 (1753)

LEON: León 30TTN91. 25-VII-1984, (LEB 26418). Leg.: F. Gómiz. En baldío.

No citada hasta el momento para la provincia de León.

Brassica barrelieri (L.) Janka, Term. Füz. 6:179 (1882)

LEON: Villar del Monte 29TQG29. 18-VI-1986, (LEB 33317). Leg.: A. Penas, M.E. García \& L. Herrero. En comunidades de Spergulario-Arnoseridetum minimae trisetetosum ovati A. Penas \& al. 1987.

Endemismo de la Península Ibérica citado por primera vez para la provincia por F. Llamas (1934) en Destriana.

Alchemilla lapeyrousii Buser, Bull. Herb. Boiss. 1, App. 2:18 (1893)

LEON: Puerto de San Isidro 30TUN07. 22-VIII-1985, (LEB 30975). Leg.: A. Penas, M.E. García \& L. Herrero. En comunidades de la Moliniorrheñatheretea R.Tx. 1937.

Citada profusamente para la vertiente septientrional de la Cordillera Cantábrica, en las vecinas provincias de Asturias y Santander. En León existe una única mención provincial (cf. E. Puente, C. Pérez \& M.J. López, 1984) de Cabornera.

Lotus tenuis Waldst. \& Kit.ex Willd., Enum. Pl. Hort. Berol. 797 (1809) var. tenuis

LEON: Cortiguera 29TPH81. 5-VIII-1985, (LEB 33138) y Bembibre 29TQH12, 5-VIII-1985, (LEB 33137). Leg.: A. Penas, M.E. García \& L. Herrero.

No citada hasta el momento de la provincia. El monógrafo A. Fernandes (1981) no recoge tampoco ninguna mención para León.

Tilia cordata Miller, Gard. Dict. ed. 8, no. 1 (1768)

LEON: Cordiñanes 30TUN48. 6-IX-1986, (LEB 33136). Leg.: A. Penas, M.E. García \& L. Herrero. En bosques del Carpinion (Issler 1931) Oberdorfer 1953.

Amo y Mora (1878) señala esta especie de los "bosques de la Región Cantábrica". No conocemos de ella ninguna mención provincial en la bibliografía consultada.

Malva nicaeensis All., Fl. Pedem. 2:40 (1785)

LEON: Lago de Carucedo 29TPH80. 6-VIII-1985, (LEB 33135). Leg.: A. Penas, M.E. García \& L. Herrero, y Valencia de Don Juan 30TTM98. 3-VI-1976 y 16-VI-1976 (LEB 6326/6327). Leg.: A. Penas.

Citada de Villafranca del Bierzo por Lange (cf. Willkomm \& Lange 1880), nuestras menciones confirman su presencia en León.

Hypericum androsaemum L., Sp. Pl. 784 (1753)

LEON: Puerto de Lumeras 29TPH84. 30-VIII-1985, (LEB 33134). Leg.: A. Penas, M.E. García \& L. Herrero. En bosques del Carpinion (Issler 1931) Oberdorfer 1953.

Escasa en la localidad herborizada, que constituye la primera cita provincial. 
Stachys heraclea All., Fl. Pedem. 1:31 (1785)

LEON: Puerto de Monteviejo 30TUn45. 21-VI-1986, (LEB 31839). Leg.: A. Penas, M.E. García \& L. Herrero.

Tercera mención provincial para esta especie que se desarrolla poco abundantemente en las localidades citadas de Puerto de Pinos (cf. C. Romero, 1983) y Cueto Ancino (cf. J. Borja, 1952). La nuestra constituye la más oriental de la provincia.

Stachys recta L., Mantissa 82 (1767) subsp. recta

LEON: Santa Marina de Valdeón 30TUN37. 6-IX-1986, (LEB 33122) y Riaño 30TUN35. 23-VI-1985. (LEB 33123). Leg.: A. Penas, M.E. García \& L. Herrero. En pedregal de río.

Confirmamos la presencia de este taxon en el Subsector Picoeuropeano. Anteriormente había sido citado por T.M. Losa (1941, 1957) en Riaño y Portilla de la Reina.

Thymus x sennenii Pau, Bol. Soc. Aragonesa Ci. Nat. 6: 29 (1907)

LEON: Geras de Gordón 30TTN75. 9-VII-1986, (LEB 33124/33125). Leg.: A.

Penas, M.E. García \& L. Herrero. En glera caliza de la orilla del río.

Híbrido citado en pocas ocasiones, del que el monógrafo R. Morales (1986) recoge una única cita en Bugedo (Burgos). Conocemos las de T.M. Losa y P. Montserrat (1951), T.M. Losa (1957) y M. Laínz (1954) de la provincia de Palencia y la de A. Caballero (1945) en la Serranía de Cuenca.

Mentha aquatica L. x M. pulegium L.

LEON: Valdefuentes '30TTM68. 4-IX-1985, (LEB 30976). Leg.: A. Penas, M.E. García \& L. Herrero.

En la bibliografía consultada no se señala el nombre que debería de llevar este taxon, del que no conocemos citas ni en León ni en las provincias limitrofes.

Verbascum blattaria L., Sp. Pl. 178 (1753)

LEON: Jabares de los Oteros 30TTM99. 24-VI-1986, (LEB 32100). Leg.: A. Penas, M.E. García \& L. Herrero.

Conocida de alguna de las provincias limítrofes, constituye la primera mención provincial.

Veronica austriaca L. subsp. vahlii (Gaudin) D.A. Webb, Bot. Jour. Linn. Soc. 65:267 (1972)

LEON: Puerto de la Magdalena 29TQH24. 13-VII-1986, (LEB 33139). Leg.: A. Penas, M.E. García \& L. Herrero. En lugares húmedos.

Para la provincia de León aportamos la cita más occidental de este taxon, del que ya conocíamos dos localidades: Riaño y Vegamián (cf. M. Laínz, 1976).

Inula salicina L. subsp. aspera (Poiret) Hayek, Prodr. Fl. Penins. Bal. 2:602 (1931).

LEON: Santovenia del Monte 30TTN92. 16-VIII-1985, (LEB 33132) y Pantano del Porma 30TUN15. 25-VII-1986, (LEB 33131). Leg.: A. Penas, M.E. García \& L. Herrero.

Primera mención provincial.

Anthemis arvensis L. subsp. incrassata (Loisel.) Nyman, Consp. 361 (1879) LEON: Foncebadón 29TQH10. 9-IX-1985, (LEB 33130), S. Félix de Valdería 29TQG47. 4-IX-1985, (LEB 33i29) y Candín 29TPH84. 30-VIII-1985, (LEB 33128). Leg.: A. Penas, M.E. García \& L. Herrero. En comunidades de la Ruderali-Secalietea Br.-Bl. 1936.

Taxon mediterráneo no citado anteriormente de la provincia de León. 
Carduus bourgeanus Boiss. \& Reuter, Pugillus 62 (1852)

LEON: Valencia de Don Juan 30TTM98. 24-VI-1986, (LEB 32133). Leg.: A. Penas, M.E. García \& L. Herrero. En cardales de la Onopordetea acanthii Br.-Bl. 1964 em. Rivas-Martínez 1987.

Picnomon acarna (L.) Cass. Dict. Sci. Nat. 40:188 (1826)

LEON: Monte de los Asturianos (Valencia de Don Juan) 30TTM98. 21-IX-1986, (LEB 33127). Leg.: A. Penas, M.E. García \& L. Herrero. En cardales de la Onopordeted acanthii Br.-Bl. 1964 em. Rivas-Martínez 1987.

Onopordum nervosum Boiss., Voy. Bot. Midi Esp. 2:357 (1841)

LEON: Valencia de Don Juan 30TTM98. 24-VI-1986, (LEB 32139). Leg.: A. Penas, M.E. García \& L. Herrero.

Taxon típicamente mediterráneo cuya cita más norteña conocida por nosotros era Salamanca (cf. M. Laínz, 1956). Aportamos pues la más noroccidental y la primera para la provincia.

Centaurea aspera L., Sp. Pl. 916 (1753) subsp. aspera

LEON: Ponferrada 29TPH91, 13-VIII-1986, (LEB 33126) y Joarilla de las Matas 30TUM28. 24-VI-1986, (LEB 32143). Leg.: A. Penas, M.E. García \& L. Herrero. En cardales de la Onopordetea acanthii Br.-Bl. 1964 em. Rivas-Martínez 1987.

Taxon no muy citado en alguna de las provincias limítrofes y que constituye novedad principal.

Milium vernale Bieb., F1. Taur.-Cauc. 1:53 (1808)

LEON: Carucedo del Bierzo 29TQH80, 1-V-1983, (LEB 15431/15432). Leg.: A. Penas \& als.

R. Carbó, M. Mayor, J. Andrés \& J.M. Losa (1977) la mencionan por primera vez para la provincia en Brañuelo (¿Brañuelas?), sin indicar plego de herbario.

\section{BIBLIOGRAFIA}

AMO Y MORA, M. DEL -1871/1873- Flora fanerogámica de la Península Ibérica. 6 vol. Imp. I, Ventura, Granada.

CABALlero, A. -1945- Apuntes para una flórula de la Serranía de Cuenca, Anales Jard. Bot. Madrid VI(2):549-592.

CARBO, R., MAYOR, M., ANDRES, J. \& LOSA, J.M. -1977- Aportaciones al catálogo florístico de la provincia de León. II. Acta Bot. Malacitana, 3:63-120.

COLMEIRO, M. -1888- Enumeración y revisión de las plantas de la Península hispano-lusitana e Islas Baleares. Imp. Vạ e $\mathrm{H}^{a}$ de Fuentenebro. Madrid.

FERNANDES, A. -1981- Contribution à la connaissance des lotiers du groupe corniculatus de la Peninsule Ibérique et des îles Baleares. Bol. Soc. Brot., ser. 2, 55:29-86.

LAINZ, M. -1954- Contribución al catálogo de la flora palentina. Collect. Bot. (Barcelona), 4:82-123.

LAINZ, M. -1956- Contribución al catálogo de la flora salmantina. Anales Inst. Bot. Cavanilles, 13:469-498.

LAINZ, M. -1970- Aportaciones al conocimiento de la flora cántabro-astur IX. Bol. Inst. Est. Asturianos ser, C. 15:3-45.

LAINZ, M. -1976- Aportaciones al conocimiento de la flora cántabro-astur XI. Bol. Inst. Est. Asturianos ser, C. 22:3-44.

LAZARO IBIZA, B. -1920/1921- Compendio de la Flora Española, 3 vol. Imp. Clásica española. Madrid. 
LLAMAS, F. -1984- Flora y Vegetación de la Maragatería (León). Inst. Fray Bernardino de Sahagún. Excma. Dip. Prov. León.

LOSA, T.M. -1941- Plantas de los alrededores de Riaño (León). Anales Jard. Bot. Madrid, 2:172-188.

LOSA, T.M. -1957- Catálogo de las plantas que se encuentran en los montes palentino-leoneses. Anales Inst. Bot. Cavanilles, 15:243-376.

LOSA, T.M. \& MONTSERRAT, P. -1951- Aportación al estudio de la Flora de los Montes Cantábricos. Anales Inst. Bot. Cavanilles, 10(2):423-510.

MERINO, P.B. -1905- Flora de Galicia. 3 vol. Tipografía Galaica. Santiago de Compostela.

MORALES VALVERDE, R. -1986- Taxonomía de los géneros Thymus (excluída la sección serpyllum) y Thymbra en la Península Ibérica. Ruizia, 3.

PUENTE GARCIA, E., PEREZ MORALES, C. \& LOPEZ PACHECO, M.J. -1984- Nuevos datos para la flora leonesa. Lazaroa, 6:297-299.

ROMERO, C. -1983- Flora y vegetación de la cuenca del río Luna (León). I.C.O.N.A. Monografías 29. Ministerio de Agricultura. Madrid.

TUTIN, P.G. \& al. -1964- Flora Europaea. I, Cambridge University Press.

WILLKOMM, M. \& LANGE, J.M.C. -1870/1880- Prodromus Florae Hispanicae. 3 vol. E. Schweizebart'sche Verlagsbuchhandlung. Stuttgart.

Dirección de los autores: Departamento de Biología Vegetal (Botánica). Facultad de Biología. Universidad de León.

\title{
CONTRIBUCIONES AL CONOCIMIENTO DE LA FLORA DEL NW DE MURCIA. II.
}

\author{
Caridad SELMA \& Oswaldo SOCORRO
}

RESUMEN: Se incluye en este trabajo las citas de 26 taxones del NW de la provincia de Murcia, de las que 24 de ellas resultan ser nuevas para la provincia.

SUMMARY: In this paper 26 taxa from NW of province Murcia are reported, 24 of these taxa are new records for this province.

Continuamos con esta nota la serie emprendida anteriormente Selma \& Socorro (1985). Con ellas queremos dar a conocer citas nuevas o criticas, o bien confirmar las ya existentes para la flora tanto del $\mathrm{NW}$ como a nivel provincial. Ello es el 